\title{
Inheritance of Persistent-Green Color in Asparagus Officinalis, L. ${ }^{1}$
}

\author{
H. Irizarry, ${ }^{2}$ J. Howard Ellison, and Portia Orton ${ }^{3}$
}

\section{INTRODUCTION}

This study deals with the inheritance of a presumed spontaneous mutation that occurred in asparagus, in which the mutants, termed "persistentgreen", have dark-green stalks and foliage in the autumn when normal plants turn yellow and brown. The persistent-green plants remained green until killed by freezing. The two persistent-green plants discovered in 1959 were from the Viking strain. This type of variant is of extremely low occurrence, in that the authors have surveyed approximately 500,000 plants of commercial Washington fields without finding more than 5 such plants.

Literature reports of the origin and genetics of plant color variations reveal that pigmentation changes usually seem to originate as spontaneous mutations and are conditioned by a single gene $(1,3,4,9) \cdot{ }^{4}$ One report indicated that a color variant was controlled by two nonallelic recessive genes (8).

A secondary part of this study was to determine the effect of the color gene or genes on the plant pigment system by means of spectrophotometric analyses. Similar studies have been reported in other crops $(5,6,9)$.

An attempt also was made to identify the persistent-green mutant in the seedling stage, as has been reported in tomato, corn, and castorbean $(1,2,7)$.

\section{MATERIAL AND METHODS}

\section{INHERITANCE STUDIES}

During the winter of 1962-63, at Rutgers University, New Jersey, a cross was made between the two persistent-green parents, V15 9 and V17 $\sigma^{7}$. These parents also were crossed with normal plants, RR 27 우, RR 29 우, and RR $35 \sigma^{7} . F_{1}$ seeds were planted in the field during the spring of 1963,

${ }_{1}^{1}$ Thesis work submitted to the Graduate School of Rutgers-The State University, New Brunswick, N.J., in partial fulfillment of the requirements for the degree of Master of Science.

${ }^{2}$ Assistant Plant Breeder, Agricultural Experiment Station, University of Puerto Rico, Río Piedras, P.R.

${ }^{3}$ Professor and Research Specialist in Vegetable Crops, and Senior Laboratory Technician, respectively, Rutgers-The State University, New Brunswick, N.J. The authors are grateful for the assistance and advice rendered by Dr. C. Price, of the Plant Biology Department, concerning the pigment studies.

* Italic numbers in parentheses refer to Literature Cited, p. 78. 
and the progenies were classified as either persistent-green or normal in the autumn.

From this planting, the following $\mathrm{F}_{1}$ progenies were selected for further study of the $\mathrm{F}_{2}$ and reciprocal $\mathrm{BC}_{1}$ generations: $\mathrm{V} 15 \times \mathrm{V} 17\left(\mathrm{~F}_{2}\right.$ only), $\mathrm{V} 15 \times \mathrm{RR} 35$, RR $27 \times \mathrm{V} 17$, and RR $29 \times \mathrm{V} 17$. The latter progeny segregated for persistent-green phenotype and both normal and persistentgreen plants were selected for study.

During the winter of $1963-64, \mathrm{~F}_{2}$ crosses and reciprocal $\mathrm{BC}_{1}$ were made in the greenhouse. In June, 1964, 100 seeds of each cross were planted in the field. A segregation count based on the foliage color was recorded for each cross and backcross during October 1964.

\section{PLANT PIGMENT STUDIES}

In July, 1964, $1 \mathrm{~g}$. of fresh plants tissue was collected from a shoot of a persistent-green and normal parent for pigment analysis. These plants were growing under similar field conditions and the stalks were approximately of the same age. Each individual plant sample was treated with methanol for the extraction of the chlorophyll and carotene. This was followed by recording an absorption spectrum curve of the 50-percent diluted extract, using a 1-mm. light path at a wavelength of 700 to $400 \mathrm{~m} \mu .^{5}$

A second pigment sample was taken in October 1964, when the normal plants had developed a yellow color and the persistent-green plants remained green.

\section{IDENTIFICATION OF PERSISTENT-GREEN PLANTS \\ IN THE SEEDLING STAGE}

As a preliminary test, $10 \mathrm{~F}_{1}$ seeds each of $\mathrm{V} 15 \times \mathrm{V} 17, \mathrm{RR} 27 \times \mathrm{V} 17$, and RR $29 \times$ V17 were sown in sand in the greenhouse during the winter of 1963-64. In the summer of 1964,100 seeds each of $\mathrm{F}_{2}$ of V15 $\times \mathrm{V} 17$, as well as $\mathrm{F}_{2}$, and reciprocal $\mathrm{BC}_{1}$ generations of $\mathrm{V} 15 \times \mathrm{RR} 35$, also were sown in the greenhouse. Water was supplied daily and a complete nutrient solution was applied twice a week for 4 weeks, after which the nutrient solution was withheld to allow yellowing of the normal plants. After 6 weeks, each seedling was identified as either persistent-green (green foliage) or normal (yellow foliage).

\section{RESULTS AND DISCUSSION}

\section{INHERITANCE STUDIES}

On the basis of the analyses of the $\mathrm{F}_{1}, \mathrm{~F}_{2}$, and reciprocal $\mathrm{BC}_{1}$ generations, persistent-green color in asparagus apparently is inherited as a single re-

${ }^{5}$ Beckman, D. B., Spectrophotometer used for absorption spectrum curve. 
cessive gene (tables $1,2,3$ ). All the segrating progenies gave $\mathrm{X}^{2}$ values in which the observed ratios fit similar expected ratios.

It is evident that asparagus plant RR 29 (normal phenotype) is heterozygous for the described character. This might suggest that the persistentgreen homozygous recessive mutant can be used for the identification of heterozygous genotypes.

TABLE 1.-F $F_{1}$ segregation of persistent-green character in asparagus

\begin{tabular}{|c|c|c|c|c|c|c|c|}
\hline \multirow[b]{2}{*}{$\begin{array}{l}P_{1} \text { females } \\
\times P_{1} \text { males }\end{array}$} & \multirow[b]{2}{*}{ Phenotypes } & \multirow[b]{2}{*}{ Tested ratios } & \multicolumn{2}{|c|}{$\begin{array}{l}\text { Classification of } \\
\text { F } 1 \text { progenies }^{2}\end{array}$} & \multirow[b]{2}{*}{ Total } & \multirow[b]{2}{*}{$\mathrm{X}^{2}$} & \multirow[b]{2}{*}{$\mathrm{P}$} \\
\hline & & & $\begin{array}{c}\text { Number } \\
\text { of } \\
\text { normal } \\
\text { pheno- } \\
\text { types }\end{array}$ & $\begin{array}{c}\text { Number } \\
\text { of per- } \\
\text { sistent- } \\
\text { green } \\
\text { pheno- } \\
\text { types }\end{array}$ & & & \\
\hline $\begin{array}{l}\mathrm{V} 15 \\
\times \mathrm{V} 17\end{array}$ & $\begin{array}{c}\text { Persistent- } \\
\text { green } \\
\text { do. }\end{array}$ & $\begin{array}{l}\text { All persist- } \\
\text { ent-green }\end{array}$ & 0 & 30 & 30 & 一 & - \\
\hline V15 & $\begin{array}{l}\text { Persistent- } \\
\text { green }\end{array}$ & All normal & 30 & 0 & 30 & - & - \\
\hline × RR35 & Normal & & & & & & \\
\hline $\begin{array}{l}\text { RR27 } \\
\times \quad V 17\end{array}$ & $\begin{array}{l}\text { Normal } \\
\text { Persistent- } \\
\text { green }\end{array}$ & do. & 30 & 0 & 30 & - & 一 \\
\hline $\begin{array}{l}\text { RR29 } \\
\times \quad \text { V17 }\end{array}$ & $\begin{array}{l}\text { Normal }^{1} \\
\text { Persistent- } \\
\text { green }\end{array}$ & $1: 1$ & 13 & 17 & 30 & 0.534 & $0.30-0.50$ \\
\hline
\end{tabular}

${ }^{1}$ Heterozygous.

PLANT PIGMENT STUDIES

Little difference in quantity of pigments between persistent-green and normal plants was found in July (fig. 1). At this stage, identification of the persistent-green plants would be difficult, if not impossible. Near the end of the growing season (October) both chlorophyll and carotene concentrations were much greater in the persistent-green mutant (fig. 1). In the fall, persistent-green and normal plants can be identified visually or by pigment analysis.

No apparent qualitative differences were observed between persistentgreen and normal plant-pigment systems (fig. 1). However, similar studies conducted by Inman et al. (5) indicated that differences did exist in Datura. 


\section{IDENTIFICATION OF PERSISTENT-GREEN PLANTS \\ IN THE SEEDLING STAGE}

The genetic ratios obtained from the greenhouse classification of asparagus seedlings as either persistent-green (green foliage) or normal (yellow foliage), are in accordance with those previously recorded in the field, using the same seed stocks (table 4). Withholding nutrients from

TABLE 2.- $F_{2}$ segregation of persistent-green character in asparagus

\begin{tabular}{|c|c|c|c|c|c|c|c|}
\hline \multirow[b]{2}{*}{$\begin{array}{l}F_{1} \text { generation } \\
\text { siblings }\end{array}$} & \multirow[b]{2}{*}{ Phenotypes } & \multirow[b]{2}{*}{$\begin{array}{l}\text { Expected } \\
\text { ratios }\end{array}$} & \multicolumn{2}{|c|}{$\begin{array}{l}\text { Classification of } \\
F_{2} \text { progenies }\end{array}$} & \multirow[b]{2}{*}{ Total } & \multirow[b]{2}{*}{$\mathrm{X}^{2}$} & \multirow[b]{2}{*}{$\mathrm{P}$} \\
\hline & & & $\begin{array}{c}\text { Number } \\
\text { of } \\
\text { normal } \\
\text { pheno- } \\
\text { types }\end{array}$ & $\begin{array}{l}\text { Number } \\
\text { of per- } \\
\text { sistent- } \\
\text { green } \\
\text { pheno- } \\
\text { types }\end{array}$ & & & \\
\hline $\begin{array}{l}(\mathrm{V} 15 \times \mathrm{V} 17) \\
\times(\mathrm{V} 15 \times \mathrm{V} 17)\end{array}$ & $\begin{array}{l}\text { Persistent- } \\
\text { green }\end{array}$ & $\begin{array}{l}\text { All persist- } \\
\text { ent-green }\end{array}$ & 0 & 59 & 59 & - & - \\
\hline $\begin{array}{l}(\mathrm{V} 15 \times \mathrm{RR} 35) \\
\times(\mathrm{V} 15 \times \mathrm{RR} 35)\end{array}$ & Normal & $3: 1$ & 38 & 6 & 44 & 3.031 & $0.05-0.10$ \\
\hline $\begin{array}{l}(\mathrm{RR} 27 \times \mathrm{V} 17) \\
\times(\mathrm{RR} 27 \times \mathrm{V} 17)\end{array}$ & do. & $3: 1$ & 41 & 12 & 53 & .159 & $.50-0.70$ \\
\hline $\begin{array}{l}(\mathrm{RR} 29 \times \mathrm{V} 17) \\
\times(\mathrm{RR} 29 \times \mathrm{V} 17)\end{array}$ & do. & $3: 1$ & 33 & 15 & 48 & 1.000 & $.30-0.50$ \\
\hline $\begin{array}{l}(\mathrm{RR} 29 \times \mathrm{V} 17) \\
\times(\mathrm{RR} 29 \times \mathrm{V} 17)\end{array}$ & $\begin{array}{l}\text { Persistent- } \\
\text { green }\end{array}$ & $\begin{array}{l}\text { All persist- } \\
\text { ent-green }\end{array}$ & 0 & 44 & 44 & - & - \\
\hline
\end{tabular}

seedlings in sand culture in the greenhouse produced color changes similar to those observed in the field in autumn.

\section{SUMMARY}

Two mature, dark-green asparagus plants (one female and one male) termed "persistent-green" were selected in a New Jersey asparagus field on November 11, 1959, when the other plants were yellow or brown. The two persistent-green plants were crossed; each of them was crossed also with normal plants for the genetic study of this character.

A secondary part of this study was to determine the effect of the color gene or genes on the plant-pigment system by means of spectrophotometric analyses. 


\begin{tabular}{|c|c|c|c|c|c|c|c|}
\hline & & & $\begin{array}{c}\text { Classific } \\
\mathrm{BC}_{1} \mathrm{pr}\end{array}$ & $\begin{array}{l}\text { ation of } \\
\text { ogenies }\end{array}$ & & & \\
\hline $\begin{array}{l}\mathrm{P} \text { and } \mathrm{F}_{1} \\
\text { parents }\end{array}$ & Phenotypes & $\begin{array}{l}\text { Expected } \\
\text { ratios }\end{array}$ & $\begin{array}{c}\text { Number } \\
\text { of } \\
\text { normal } \\
\text { pheno- } \\
\text { types }\end{array}$ & \begin{tabular}{|} 
Number \\
of per- \\
sistent- \\
green \\
pheno- \\
types
\end{tabular} & Total & $\mathrm{X}^{2}$ & \\
\hline $\begin{array}{l}(\mathrm{V} 15 \times \mathrm{RR} 35) \\
\mathrm{BC} \text { RR35 }\end{array}$ & $\begin{array}{r}\text { Normal } \\
\text { do. }\end{array}$ & $\begin{array}{l}\text { All } \\
\text { normal }\end{array}$ & 51 & 0 & 51 & 一 & - \\
\hline V15 & $\begin{array}{l}\text { Persistent- } \\
\text { green }\end{array}$ & $1: 1$ & 26 & 31 & 57 & 0.438 & $0.50-0.70$ \\
\hline $\mathrm{BC}(\mathrm{V} 15 \times \mathrm{RR} 35)$ & Normal & & & & & & \\
\hline $\begin{array}{l}(\mathrm{R} R 27 \times \mathrm{V} 17) \\
\mathrm{BC} \mathrm{V17}\end{array}$ & $\begin{array}{c}\text { do. } \\
\text { Persistent- } \\
\text { green }\end{array}$ & $1: 1$ & 33 & 37 & 70 & .228 & $.50-0.70$ \\
\hline $\begin{array}{l}\mathrm{RR} 27 \\
\mathrm{BC}(\mathrm{RR} 27 \times \mathrm{V} 17)\end{array}$ & $\begin{array}{r}\text { Normal } \\
\text { do. }\end{array}$ & $\begin{array}{l}\text { All } \\
\text { normal }\end{array}$ & 93 & 0 & 93 & - & - \\
\hline $\begin{array}{l}(\mathrm{R} R 29 \times \mathrm{V} 17) \\
\mathrm{BC} \text { V17 }\end{array}$ & $\begin{array}{l}\text { do. } \\
\text { Persistent- } \\
\text { green }\end{array}$ & $1: 1$ & 20 & 29 & 49 & 1.654 & $.10-0.20$ \\
\hline $\begin{array}{l}(\mathrm{RR} 29 \times \mathrm{V} 17) \\
\mathrm{BC} \text { V17 }\end{array}$ & $\begin{array}{l}\text { do. } \\
\text { do. }\end{array}$ & $\begin{array}{l}\text { All } \\
\text { persistent- } \\
\text { green }\end{array}$ & 0 & 46 & 46 & 一 & - \\
\hline $\begin{array}{l}\text { RR29 } \\
\text { BC }(\operatorname{RR} 29 \times V 17)\end{array}$ & $\begin{array}{l}\text { Normal }^{1} \\
\text { Persistent- } \\
\text { green }\end{array}$ & $1: 1$ & 18 & 14 & 32 & .500 & $.30-0.50$ \\
\hline $\begin{array}{l}\mathrm{RR} 29 \\
\mathrm{BC}(\mathrm{RR} 29 \times \mathrm{V} 17)\end{array}$ & $\begin{array}{c}\text { Normal }^{1} \\
\text { do. }\end{array}$ & $3: 1$ & 22 & 12 & 34 & 1.921 & $.10-0.20$ \\
\hline
\end{tabular}

\footnotetext{
${ }^{1}$ Heterozygous.
}

An attempt also was made to identify the persistent-green mutants in the seedling stage.

The study of the phenotypes of $17 \mathrm{~F}_{1}, \mathrm{~F}_{2}$, and reciprocal $\mathrm{BC}_{1}$ progenies indicated that persistent-green color in asparagus is inherited as a single recessive gene.

There was a large quantitative difference in chlorophyll and carotene between the persistent-green and normal plant complexes in October, but 
not in July. Apparently the persistent-green mutants retain chlorophyll and carotene much later in the season than do the normal plants. No qualitative difference in pigment was found in either July or October.
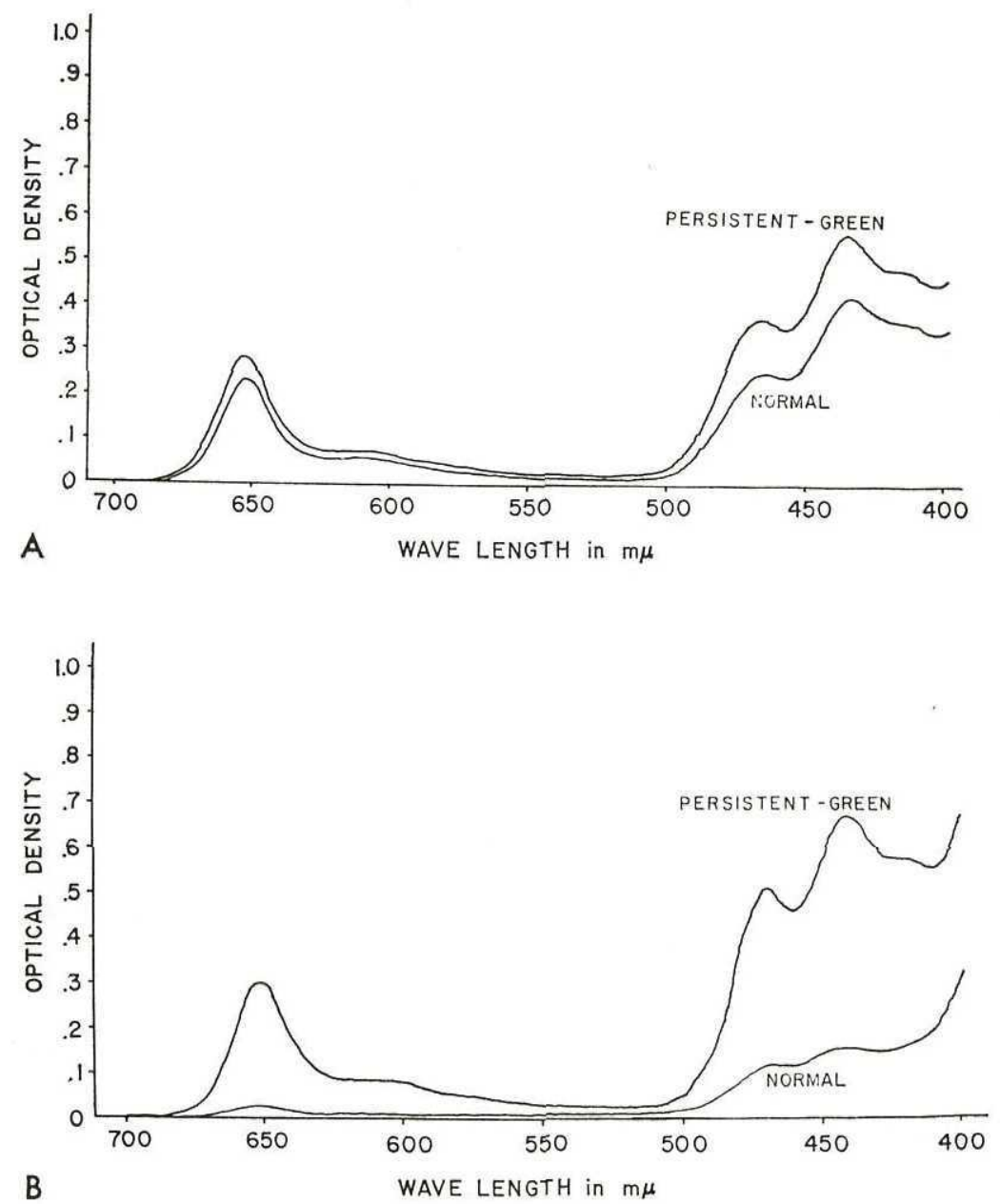

FIG. 1-Absorption spectrum of the 50-percent diluted extracts from a persistentgreen and a normal asparagus plant, using 1-mm. light path. A, Plants sampled in July; B, plants sampled in October.

Asparagus seedlings were easily classified as to persistent-green (green foliage) or normal (yellow foliage) in the greenhouse when the plants were 6 weeks old. 


\section{RESUMEN}

El 11 de noviembre, cuando el follaje de las demás plantas era amarillo o pardo, se seleccionaron en un campo de New Jersey dos plantas de espárrago

TABLE 4.- $F_{1}, F_{2}$, and reciprocal $B C_{1}$ segregation of persistent-green character in asparagus, grown under greenhouse conditions

\begin{tabular}{|c|c|c|c|c|c|c|c|}
\hline \multirow[b]{2}{*}{$P_{1}$ and $F_{1}$ parents } & \multirow[b]{2}{*}{ Phenotypes } & \multirow[b]{2}{*}{ Expected ratios } & \multicolumn{2}{|c|}{$\begin{array}{l}\text { Classification of } \\
F_{1}, F_{2}, \text { and } \\
\text { BC1 progenies }\end{array}$} & \multirow[b]{2}{*}{ Total } & \multirow[b]{2}{*}{$\mathrm{X}^{2}$} & \multirow[b]{2}{*}{$\mathrm{P}$} \\
\hline & & & $\begin{array}{l}\text { Num- } \\
\text { ber of } \\
\text { normal } \\
\text { pheno- } \\
\text { types }\end{array}$ & \begin{tabular}{|c} 
Num- \\
ber of \\
persist- \\
ent- \\
green \\
pheno- \\
types
\end{tabular} & & & \\
\hline $\begin{array}{l}\mathrm{V} 15 \\
\times \mathrm{V} 17^{1}\end{array}$ & $\begin{array}{c}\text { Persistent- } \\
\text { green } \\
\text { do. }\end{array}$ & $\begin{array}{l}\text { All } \\
\text { persistent- } \\
\quad \text { green }\end{array}$ & 0 & 10 & 10 & - & - \\
\hline $\begin{array}{l}\text { RR } 27 \\
\times \quad V 17^{1}\end{array}$ & $\begin{array}{l}\text { Normal } \\
\text { Persistent- } \\
\text { green }\end{array}$ & $\begin{array}{l}\text { All } \\
\text { normal }\end{array}$ & 10 & 0 & 10 & - & - \\
\hline $\begin{array}{l}\text { RR } 29 \\
\times \quad V 17^{1}\end{array}$ & $\begin{array}{l}\text { Normal }^{2} \\
\text { Persistent- } \\
\text { green }\end{array}$ & $1: 1$ & 9 & 7 & 16 & 0.250 & $0.50-0.70$ \\
\hline $\begin{array}{l}(\mathrm{V} 15 \times \mathrm{V} 17) \\
\times(\mathrm{V} 15 \times \mathrm{V} 17)^{3}\end{array}$ & do. & $\begin{array}{l}\text { All } \\
\text { persistent- } \\
\text { green }\end{array}$ & 0 & 100 & 100 & - & - \\
\hline $\begin{array}{l}(\mathrm{V} 15 \times \mathrm{RR} 35) \\
\times(\mathrm{V} 15 \times \mathrm{RR} 35)^{3}\end{array}$ & Normal & $3: 1$ & 56 & 21 & 77 & .212 & $.50-0.70$ \\
\hline $\begin{array}{l}\left(\mathrm{V} 15 \underset{\mathrm{BC}}{\times} \mathrm{RR} 35^{3}\right. \\
\text { BR35) }\end{array}$ & $\begin{array}{l}\text { do. } \\
\text { do. }\end{array}$ & $\begin{array}{l}\text { All } \\
\text { normal }\end{array}$ & 91 & 0 & 91 & - & - \\
\hline
\end{tabular}

${ }^{1}$ First experiment, winter 1963-64.

${ }^{2}$ Heterozygous.

${ }^{3}$ Second experiment, summer 1964.

ㅇ y $\sigma^{\nearrow}$, cuyo follaje era de un color verde obscuro, el cual se designó como "verde-persistente".

Para estudiar este carácter genético las dos plantas se cruzaron entre sí y luego también se cruzó cada una con otras plantas de color normal.

El estudio tuvo como propósito secundario el determinar mediante el uso del espectrofotómetro, el efecto que el gene o genes responsables del 
color puedan tener sobre el sistema de pigmentación de las plantas de espárrago.

También se trató de identificar en plántulas en el invernadero los mutantes de color verde-persistente.

El estudio del fenotipo de 17 progenies $\mathrm{F}_{1}, \mathrm{~F}_{2}$ y del retrocruce ${ }_{1}$, indicó que el color verde-persistente en el follaje de la planta de espárrago se hereda como un gene recesivo simple.

Se observó una gran diferencia cuantitativa de clorofila y carotina entre las plantas de follaje verde-persistente y las de color normal en el mes de octubre, pero no en julio. Aparentemente, los mutantes retienen la clorofila y la carotina hasta más tarde en el otoño que las plantas de color normal. No se observaron diferencias cualitativas entre los pigmentos en ambos tipos de planta durante julio u octubre.

Fue fácil diferenciar entre las plantas de espárrago de color verdepersistente (follaje verde) y las de color normal (follaje amarillo) en el invernadero cuando tenían 6 semanas de nacidas.

\section{LITERATURE CITED}

1. Andrus, C. F., A character useful in the study of natural eross-pollination in tomatoes, Proc. Amer. Soc. Hort. Sci. 57: 345-6, 1951.

2. Demerec, M., Inheritance of pale-green seedlings in maize, Genetics 10: 318-44, 1925.

3. Emerson, R. A., The Inheritance of certain Forms of Chlorophyll Reduction in Corn Leaves, Nebraska Agr. Expt. Sta. Ann. Rept. 25, 89-105, 1912.

4. Highkin, H. R., Chlorophyll studies on barley mutants, Plant Physiol. 25: 294-306, 1950 .

5. Inman, O. L., and Blakeslee, A. F., New or modified chlorophylls resulting from a recessive pale mutation in Datura, Science (abs.) 87: 428-9, 1938.

6. Lesley, J. W., Lesley, M. M., and Turrell, F. M., Cytogenetic and pigment studies of blue-green mutant from P32-treated seeds of the tomato, Amer. J. Bot. 45: 598-602 1958.

7. Stein, H., Colour Inheritance in Ricinus, Bull. Res. Council of Israel (abs.) 7 (D) $113,1959$.

8. Thompson, A. E., Inheritance of high total carotenoid pigments in tomato fruits, Science 121: 896-7, 1955.

9. Whitaker, T. W., Genetic and chlorophyll studies of a yellow-green mutant in muskmelon, Plant Physiol. 27: 263-7, 1952. 